\title{
Severe rhabdomyolysis following an uncomplicated endoscopic procedure: a case report
}

\author{
Jenny J. Tang, BA, Kevin S. Park, MD, Govind R. Rajan, MD, Emily B. Goldenberg, MD \\ Department of Anesthesiology and Perioperative Care, University of California, Irvine, 333 City Boulevard West, Suite 2150, \\ Orange, CA 92868, USA.
}

Correspondence: Kevin S. Park, M.D., Department of Anesthesiology and Perioperative Care, University of California, Irvine, 333 City Boulevard West, Suite 2150, Orange, CA 92868, USA. Phone: 714-456-5501. E-mail: seungmp@hs.uci.edu.

\begin{abstract}
Rhabdomyolysis in adults after anesthetic administration is uncommon in those without underlying risk factors. We present a 34year-old female with history of severe gastroesophageal reflux disease (GERD), hypothyroidism, and migraines who developed severe rhabdomyolysis following an uncomplicated general anesthetic for esophagogastroduodenoscopy (EGD) and placement of Bravo esophageal $\mathrm{pH}$ monitor. The patient did not have any known risk factors for malignant hyperthermia, and halogenated agents were not used during the procedure. We speculate that the rhabdomyolysis was caused by the use of succinylcholine. Providers should be aware that even patients without known risk factors may be at risk for developing rhabdomyolysis, and it is important to remain vigilant about this potentially life-threatening complication.
\end{abstract}

Keywords: Rhabdomyolysis; Esophagogastroduodenoscopy; Endoscopy; Succinylcholine; Myalgia

Citation: Tang JJ, Park KS, Rajan GR, Goldenberg EB. Severe rhabdomyolysis following an uncomplicated endoscopic procedure: a case report. Anaesth. pain intensive care 2020;24(3):354-357.

Received - 24 March 2020; Reviewed \& Accepted - 27 March 2020

\section{Introduction}

Rhabdomyolysis is a medical condition defined by the rapid breakdown of damaged skeletal muscle with subsequent release of electrolytes and proteins, notably creatine kinase (CK), lactate dehydrogenase, and potassium, into the bloodstream. ${ }^{1}$ It has a variety of clinical presentations, ranging from asymptomatically increased $\mathrm{CK}$ to life-threatening acute renal failure, compartment syndrome, electrolyte imbalances, and cardiac arrest. Rhabdomyolysis has specifically been linked to the anesthetic administrations of succinylcholine, halogenated agents, and propofol, especially in certain susceptible populations. These include patients with underlying neuromuscular or mitochondrial disease, or those susceptible to malignant hyperthermia (MH).
We present a case of rhabdomyolysis following an uncomplicated general anesthesia for esophagogastroduodenoscopy (EGD) and placement of wireless Bravo esophageal $\mathrm{pH}$ monitoring device. Patient information was protected according to the institutional Health Insurance Portability and Accountability Act of 1996 (HIPPA) requirements.

\section{Case report}

A 34-year-old female with weight of $92 \mathrm{~kg}$ and body mass index of $28 \mathrm{~kg} / \mathrm{m}^{2}$ with severe gastroesophageal reflux disease (GERD), hypothyroidism, and migraines presented for elective EGD and Bravo placement. She had no drug allergies and her outpatient medications included esomeprazole, levothyroxine, and sumatriptan. She had an 
Table 1: Results of laboratory investigations

\begin{tabular}{llll}
\hline Laboratory test & Change & Patient's result & Reference value \\
\hline Phosphorus & $\downarrow$ & $2.1 \mathrm{mg} / \mathrm{dL}$ & $2.5-5.0 \mathrm{mg} / \mathrm{dL}$ \\
\hline TSH & $\uparrow$ & $6.316 \mathrm{mU} / \mathrm{L}$ & $0.540-4.120 \mathrm{mU} / \mathrm{L}$ \\
\hline Alanine aminotransferase (ALT) & $\uparrow$ & $80 \mathrm{U} / \mathrm{L}$ & $7-52 \mathrm{U} / \mathrm{L}$ \\
\hline Aspartate aminotransferase (AST) & $\uparrow \uparrow$ & $346 \mathrm{U} / \mathrm{L}$ & $13-39 \mathrm{U} / \mathrm{L}$ \\
\hline Lactate dehydrogenase & $\uparrow$ & $1515 \mathrm{U} / \mathrm{L}$ & $140-271 \mathrm{U} / \mathrm{L}$ \\
\hline CK & $\uparrow \uparrow \uparrow$ & $>20,000 \mathrm{U} / \mathrm{L}$ & $30-223 \mathrm{U} / \mathrm{L}$ \\
\hline Sodium & $\mathrm{N}$ & $140 \mathrm{mmol} / \mathrm{L}$ & $135-145 \mathrm{mmol} / \mathrm{L}$ \\
\hline Potassium & $\mathrm{N}$ & $3.6 \mathrm{mmol} / \mathrm{L}$ & $3.5-5.5 \mathrm{mmol} / \mathrm{L}$ \\
\hline Chloride & $\uparrow$ & $110 \mathrm{mmol} / \mathrm{L}$ & $98-106 \mathrm{mmol} / \mathrm{L}$ \\
\hline Bicarbonate & $\mathrm{N}$ & $23 \mathrm{mmol} / \mathrm{L}$ & $22-29 \mathrm{mmol} / \mathrm{L}$ \\
\hline Creatinine & $\uparrow$ & $1.0 \mathrm{mg} / \mathrm{dL}$ & $0.5-1.1 \mathrm{mg} / \mathrm{dL}$ \\
\hline Glucose & $\mathrm{N}$ & $82 \mathrm{mg} / \mathrm{dL}$ & $<140 \mathrm{mg} / \mathrm{dL}$ \\
\hline Blood urea nitrogen & $\mathrm{N}$ & $23 \mathrm{mg} / \mathrm{dL}$ & $8-20 \mathrm{mg} / \mathrm{dL}$ \\
\hline Urine protein & $\uparrow \uparrow$ & $100 \mathrm{mg} / \mathrm{dL}$ & 0 to $14 \mathrm{mg} / \mathrm{dL}$ \\
\hline Urine free Hb & +++ & Zero \\
\hline & & & \\
\hline
\end{tabular}

appendectomy and a caesarean section in the past without any problems related to anesthesia. Given the severity of her reflux symptoms, she underwent general anesthesia with a classic rapid sequence intubation. She was induced with fentanyl $100 \mathrm{mcg}$, lidocaine $50 \mathrm{mg}$, propofol $150 \mathrm{mg}$, and succinylcholine $80 \mathrm{mg}$ and the airway was secured utilizing classic rapid sequence intubation. Anesthesia was maintained with the propofol infusion at 250 $\mathrm{mcg} \cdot \mathrm{kg}^{-1} \cdot \mathrm{min}^{-1}$. Her vital signs remained stable throughout the procedure, which lasted approximately 30 minutes. The patient was extubated awake and her vital signs remained stable throughout her stay in post anesthesia care unit. About two hours postoperatively, the patient reported diffuse muscle aches all over her body and reported passing blood tinged urine. She further revealed that she had been experiencing intermittent, self-limiting muscle aches migrating throughout her body for the past few years. Although the clinical picture was quite confusing, we felt prudent to do a detailed laboratory workup to rule out rhabdomyolysis. Meanwhile, a fluid bolus was started, and the patient was encouraged to hydrate herself by mouth.

The results of notable laboratory studies including serum enzyme studies, urinalysis, the basic metabolic screen are given in Table 1. Additional workup was completed to assess for rheumatologic causes of rhabdomyolysis, which included rheumatoid factor, anti-nuclear antibody, anti-Jo-1 antibody, antiribonuclear protein antibody, anti-Sjögren'ssyndrome-related antibodies, and anti-mitochondrial M2 antibody (AMA). Of these, only the AMA was positive.

The patient was admitted for the management of rhabdomyolysis and treated with aggressive fluid hydration. On postoperative day three, her CK had decreased from $>20,000$ to 11,606 U/L, her AST and ALT decreased from maximum values of $602 \mathrm{U} / \mathrm{L}$ and $176 \mathrm{U} / \mathrm{L}$ on postoperative day one to $176 \mathrm{U} / \mathrm{L}$ and133 $\mathrm{U} / \mathrm{L}$, respectively, and her muscle aches had 
completely resolved. Her vital signs and other laboratory values, including serum potassium and creatinine, remained stable throughout her hospital course. She was discharged on postoperative day three with instructions to follow-up with her primary care physician in one week; however, patient declined additional follow-up.

\section{Discussion}

The gold standard for the laboratory diagnosis of rhabdomyolysis is plasma $\mathrm{CK}$ level over five times the upper limit of normal (generally around $1000 \mathrm{U} / \mathrm{L}$ ). Values above $5000 \mathrm{U} / \mathrm{L}$ are suggestive of significant muscle breakdown and increased risk for acute kidney damage. ${ }^{1}$ Our patient's presentation of severe myalgia, dark urine, and $\mathrm{CK}>20,000 \mathrm{U} / \mathrm{L}$ was highly suggestive for rhabdomyolysis. In addition, rhabdomyolysis also explains the increase in our patient's aspartate and alanine aminotransferase (AST and ALT) level, as these enzymes are also the byproduct of muscle breakdown. The higher increase in AST compared with ALT is further suggestive of ongoing damage, since AST is present in much higher concentrations in muscle.

There are a number of potential causes of rhabdomyolysis, including traumatic injuries, dysregulation of skeletal muscle calcium homeostasis such as in $\mathrm{MH}$, infections, rheumatologic diseases such as autoimmune myopathies, non-autoimmune myopathies such as Becker's muscular dystrophy, electrolyte imbalances such as hypokalemia and hypophosphatemia, and medication side effects such as statin-induced myopathy. ${ }^{2,3}$ In our patient, we did not identify any evidence of acute trauma, hypoxia, infection, or prolonged immobility, especially given the short duration of the case.

Her rheumatologic work-up was negative, except for the AMA test. The AMA is a marker that is highly specific for primary biliary cholangitis (PBC), formerly known as primary biliary cirrhosis. ${ }^{4} \mathrm{PBC}$ is a chronic autoimmune disease in which antibodies target antigens that are immunologically unique to the biliary epithelial cells of the liver. A positive AMA test with relevant clinical features, such as fatigue, pruritus, or jaundice, is highly specific for the diagnosis of PBC. Other key criteria for diagnosis include persistently elevated serum alkaline phosphatase and consistent liver histology. ${ }^{4}$ Our patient did not have any of the related clinical features or lab findings, and therefore most likely did not have PBC. While AMA does target mitochondrial antigens, a positive AMA finding or a diagnosis of PBC has not been linked to mitochondrial myopathies or myositis that may predispose patients to rhabdomyolysis. ${ }^{5}$ Similarly, there is no evidence to suggest that patients with AMA positivity or PBC are at increased risk for developing succinylcholineinduced rhabdomyolysis or propofol infusion syndrome (PRIS). This is most likely because AMA is specific to antigens of the biliary epithelial cells and is not related to the disruption of more widespread cells such as muscle. Thus, her positive AMA test result is likely an incidental finding that may place her at increased risk for future development of $\mathrm{PBC},{ }^{5}$ but probably did not play a role in the pathophysiology of her rhabdomyolysis. Therefore, the most likely culprits of our patient's rhabdomyolysis were the anesthetic medications that she received during her procedure, specifically succinylcholine and propofol.

The reported incidence of succinylcholine-induced myalgia vary widely, from $1.5 \%$ up to $89 \%$, and are usually self-limiting within 2-7 days postoperatively. ${ }^{6}$ Succinylcholine-induced rhabdomyolysis without an underlying cause in adults, on the other hand, is extremely rare. ${ }^{3}$ More often, patients who develop succinylcholine-induced rhabdomyolysis have concomitant $\mathrm{MH}$ and/or underlying neuromuscular disease. ${ }^{3}$ We considered the possibility of $\mathrm{MH}$ in our patient despite her stable perioperative and postoperative vital signs, normocarbia, and no evidence of acidosis. In our patient, the possible triggers of $\mathrm{MH}$ during the perioperative course were administration of succinylcholine and the anesthesia circuit. It is unclear what would have happened if she had undergone a longer anesthetic or received prolonged exposure to volatile agents. It is possible that $\mathrm{MH}$ can present as isolated rhabdomyolysis or myoglobinuria in otherwise asymptomatic patients. ${ }^{7}$ It additionally is not uncommon for $\mathrm{MH}$ to present in patients with negative family history and uncomplicated prior anesthetics. ${ }^{8}$ However, definitive diagnosis cannot be made without a muscle biopsy demonstrating positive response to a caffeine halothane contracture test, or identification of a recognized $\mathrm{MH}$ diagnostic mutation in either the RYR1, CACNA1S, or STAC3 genes. ${ }^{9}$ Underlying 
neuromuscular diseases, such as muscular dystrophies, can render patients susceptible to $\mathrm{MH}$ and associated rhabdomyolysis. The relevancy of this patient's intermittent, migratory muscle aches is unclear, but may increase suspicion for an underlying myopathy. These conditions, which typically present in childhood or early adulthood, remain possibilities that cannot be ruled out without comprehensive neurologic examination and genetic testing, ${ }^{3}$ which our patient has not had to our knowledge.

Rhabdomyolysis may occur after propofol administration as a component of PRIS. PRIS however has been predominantly reported in patients receiving long-term (>48 h), high dose (>5 mg/kg/h) infusions. ${ }^{10}$ Increased susceptibility to PRIS exists in populations with underlying mitochondrial disorders, although these almost always present during childhood. While our patient did receive maintenance with a relatively high dose of propofol infusion $(250 \mathrm{mcg} / \mathrm{kg} / \mathrm{min}$, or 15 $\mathrm{mg} / \mathrm{kg} / \mathrm{h}$ ), the case duration was extremely short at less than $30 \mathrm{~min}$. Moreover, the total propofol she received, $485 \mathrm{mg}$, or $5.3 \mathrm{mg} / \mathrm{kg}$, is substantially lower than the median cumulative dose of $380.4 \mathrm{mg} / \mathrm{kg}$ across previously reported cases of PRIS. ${ }^{10}$ The typical presentation of PRIS also consists of metabolic acidosis and electrocardiogram changes, which were absent in our patient. Therefore, propofol-induced rhabdomyolysis, although possible, is less likely.

\section{Conclusion}

In summary, we describe a patient who developed postoperative rhabdomyolysis after receiving succinylcholine and propofol for an uncomplicated endoscopic procedure. It is difficult to definitively identify the cause of the rhabdomyolysis, but we suspect that succinylcholine was the inciting cause. Given that other possibilities (e.g., MH, underlying mitochondrial disorder or muscular dystrophy) may still exist, it is highly recommended that such patients undergo further workup to rule in susceptibility to $\mathrm{MH}$ or presence of undiagnosed mitochondrial dysfunction. It is important for providers to remain vigilant about this potentially life-threatening complication.

\section{Conflict of interest}

None declared by the authors

\section{Authors' contribution}

JT, KP, GR, and EG: Literature review, manuscript writing

\section{References}

1. Torres PA, Helmstetter JA, Kaye AM, Kaye AD. Rhabdomyolysis: Pathogenesis, diagnosis, and treatment. Ochsner J. 2015;15(1):58-69. [PubMed]

2. Huerta-Alardin AL, Varon J, Marik PE. Bench-tobedside review: Rhabdomyolysis - an overview for clinicians. Crit Care. 2005;9(2):158-169. [PubMed] DOI: $\underline{10.1186 / \mathrm{cc} 2978}$

3. Barrons RW, Nguyen LT. Succinylcholine-induced rhabdomyolysis in adults: Case report and review of the literature. J Pharm Pract. 2020 Feb;33(1):102-107. [PubMed] DOI: $10.1177 / 0897190018795983$

4. Bowlus CL, Gershwin ME. The diagnosis of primary biliary cirrhosis. Autoimmun Rev. 2014;13(4-5):441444. [PubMed] DOI: 10.1016/i.autrev.2014.01.041

5. Yamagiwa S, Kamimura H, Takamura M, Aoyagi Y. Autoantibodies in primary biliary cirrhosis: Recent progress in research on the pathogenetic and clinical significance. World J Gastroenterol. 2014;20(10):26062612. [PubMed] DOI: $10.3748 /$ wjg.v20.i10.2606

6. Wong SF, Chung F. Succinylcholine-associated postoperative myalgia. Anaesthesia. 2000;55(2):144152. [PubMed] DOI: 10.1046/j.13652044.2000.055002144.x

7. Harwood TN, Nelson TE. Massive postoperative rhabdomyolysis after uneventful surgery: A case report of subclinical malignant hyperthermia. Anesthesiology. 1998;88(1):265-268. [PubMed] DOI: 10.1097/00000542-199801000-00036

8. Larach MG, Gronert GA, Allen GC, Brandom BW, Lehman EB. Clinical presentation, treatment, and complications of malignant hyperthermia in North America from 1987 to 2006. Anesth Analg. 2010;110(2):498-507. [PubMed] DOI: 10.1213/ANE.0b013e3181c6b9b2

9. Riazi S, Kraeva N, Hopkins PM. Malignant hyperthermia in the post-genomics era: New perspectives on an old concept. Anesthesiology. 2018;128(1):168-180. [PubMed] DOI: 10.1097/ALN.0000000000001878

10. Hemphill S, Mcmenamin L, Bellamy MC, Hopkins PM. Propofol infusion syndrome: A structured literature review and analysis of published case reports. $\mathrm{Br} J$ Anaesth. 2019;122(4):448-459. [PubMed] DOI: 10.1016/j.bja.2018.12.025 